\title{
Trends in Fuzzy Statistics
}

\author{
S. Mahmoud Taheri \\ Isfahan University of Technology, Isfahan, Iran
}

\begin{abstract}
After introducing and developing fuzzy set theory, a lot of studies have been done to combine statistical methods and fuzzy set theory. This works, called fuzzy statistics, have been developed in some branches.

In this article we review essential works on fuzzy estimation, fuzzy hypotheses testing, fuzzy regression, fuzzy Bayesian statistics, and some relevant fields.

Zusammenfassung: Nach Entwicklungen in Fuzzy Mengen-Lehre sind eine Vielfalt von Untersuchungen gemacht worden, um Stochastische Methoden und Fuzzy Mengen-Lehre zu kombinieren. Diese Arbeiten, die in verschiedenen Zweigen zu finden sind, werden mit Fuzzy-Stochastik zusammengefaßt. In dieser Arbeit geben wir eine Übersicht über Fuzzy-Abschätzungen, FuzzyTests von Hypothesen, Fuzzy-Regression, Fuzzy-Bayesianische Stochastik und einige verwandte Gebiete.
\end{abstract}

Keywords: Fuzzy Statistics, Estimation, Hypotheses Testing, Regression, Bayes Methods.

\section{Introduction}

Statistical analysis, in traditional form, is based on crispness of data, random variables, hypotheses, decision rules, parameters, and so on. As there are many different situations in which the above assumptions are rather irrealistic, there have been some attempts to analyze these situations with fuzzy set theory.

In the present paper, we try to give an overview of the combination of statistical methods and fuzzy set theory.

It should be mentioned that, we only focus on the fuzzy statistics. So, we will not consider fuzzy probability, fuzzy random variables, fuzzy stochastic processes, probabilistic interpretation of fuzzy sets, random sets approach to fuzzy sets, and so on.

With this in mind, we start in Section 2, remarking some works on statistical point estimation theory in the fuzzy environment. In Section 3, a few works on interval estimation are reviewed. Researches on fuzzy hypotheses testing are discussed in Section 4. In Section 5, we consider topic works on fuzzy regression. Section 6 contains a review of some researches on fuzzy Bayesian statistics. Finally, in Section 7, we mention some special works on pure and applied fuzzy statistics.

Let us point out two comments:

1. There are two valuable monographs by Kruse and Meyer (1987), and by Viertl (1996). In these two books, readers find attempts to unify many works in the field of descriptive statistics with fuzzy data, fuzzy random variables, statistical inference for fuzzy data, and some related fields.

2. There are a lot of discussions on the similarities and differences between probability 
(as a description of uncertainty in traditional statistics), and fuzziness. One can look in Dale (1980), Dubois and Prade (1986, 1988, 1993), Grabisch et al. (1995), Hisdal (1988), Klir (1989), Kosko (1990), Laviolette and Seaman (1992, 1994), Laviolette et al. (1995) (with discussion), Stallings (1977), and Zadeh (1995, 2002).

\section{Point Estimation}

The problem of point estimation for an unknown parameter, when there is no exact perceive of the experimental outcomes, is presented by some authors. Corral and Gil (1984) work on the problem of point estimation, and extend the maximum likelihood principle. See also Gil et al. (1985a). Gil (1987), based on the notion of fuzzy information system, investigates the uncertainty associated with inference from a fuzzy information system. They study some statistical problems, such as point estimation, with this point of view.

Kruse (1984), and Kruse and Meyer (1987) explain some methods for point and interval estimation for samples of fuzzy random variables.

Buckley (1983b) studies the problem of estimation, with fuzzy data, by a fuzzy decision making approach. In his approach, an optimal decision is a decision function from the data into the set of actions with maximum membership in some fuzzy set.

Okuda (1987) considers fuzzy observations to estimate moments and parameters of a population transforming the effect of fuzziness contained in fuzzy observations into correction terms. He discusses maximum likelihood estimators and the loss of information due to fuzziness.

With the availability of the concept of a fuzzy random variable, Yao and Hwang (1996) discuss the random samples of size $n$ with one vague observation associated with probability density function $\mathrm{f}(\mathrm{x} ; \theta)$. They describe sufficient statistic, unbiased estimator, and the information inequality under these conditions. They also define maximum likelihood estimators for unknown parameters, on the basis of a sample of fuzzy random variables (Hwang and Yao, 1996).

In a completely different approach, Manton et al. (1991), and Manton et al. (1994), provide a method of estimation from longitudinal surveys regarding fuzzy states.

Using empirical distribution function, Viertl (1996) studies some nonparametric methods in estimation using fuzzy data. Based on some results on expectation of fuzzy random variables, Lopez-Diaz and Gil (1998) derive some statistical inference methods, and study their applications specially in statistical decision theory with fuzzy losses and fuzzy utilities.

Some works have been done on the basis of the fuzzy variables in Nahmias' sense (Nahmias, 1978). For example, Cai et al. (1991) propose a method for estimating parameters of membership functions through defining a likelihood function, and study its applications in fuzzy software reliability modeling. In this context, Cai (1993) discusses the parameter estimation methods for normal membership functions.

\section{Interval Estimation}

Corral and Gil (1988) formulate the problem of interval estimation when the experimental 
outcomes cannot be exactly perceived, but they may be assimilated with fuzzy information. Gil (1992) studies the connection between fuzzy numbers and random intervals, as well.

Watanabe (1996) derives some results on fuzzy random variables from the statistical point of view, and specially studies fuzzy interval estimation.

Viertl (1996) discusses the problem of confidence regions based on fuzzy data.

It should be mentioned that, McCain (1983) uses the phrase "fuzzy confidence interval" for an alternative interpretation of fuzzy numbers, without statistical interpretation.

\section{Hypothesis Testing}

Testing of statistical hypotheses using fuzzy sets, is developed in different approaches.

Casals et al. (1986a,b), and Casals and Gil (1989) discuss statistical hypothesis testing based on a model represented by fuzzy events. They extend Neyman-Pearson Lemma and Bayes method to this case. Gil et al. (1985b) study a goodness of fit test with fuzzy observations, as well.

Saade and Schwarzlander (1990) develop fuzzy hypothesis testing for hybrid data under which one hypothesis is a mixture of a random and a fuzzy component. In their formulation the likelihood ratio, which is normally utilized in such types of decision making problems, is fuzzified and compared to a threshold.

Son et al. (1992), using a generalized Neyman-Pearson lemma, present a locally most powerful fuzzy test and study its application in signal detection.

Watanabe and Imaizumi (1993) introduce a testing method of a fuzzy hypothesis for random data. They didn't precisely define the probabilities of type I and type II errors. They have defined the generalized power function as the expected value of a fuzzy critical function. In their method, the conclusion from the test is also fuzzy.

Takayanagi and Cliff (1994) explain the human imprecision of the choice boundary in hypothesis testing. In particular, they attempt to determine how membership functions and the fuzziness index clearly show the human decision ambiguities that are reflected in formal statistical investigations.

Romer and Kandel (1995) investigate the impacts of vague data on the statistical task of hypotheses testing.

Arnold (1995, 1996, 1998), for the first time, present an approach how to test fuzzily formulated hypotheses with crisp data in which the probabilities of type I and type II errors are defined. His approach focuses on one-parameter exponential family. He studies the application of his method to some one-sided and two-sided tests. The same problem was considered by Delgado et al. (1985) with another approach.

Taheri and Behboodian (1999) formulate the problem of fuzzy hypotheses testing when the hypotheses are fuzzy and the observations are crisp. In order to establish optimality criteria, they give new definitions for probability of type I and type II errors. Then, on the basis of these new errors, they state and prove Neyman-Pearson Lemma for fuzzy hypotheses testing. Paris (2001) applies their method to analyze the effect of squeezing the channel in binary communication. 
With the help of a central limit theorem, Korner (2000) obtains an asymptotic test of hypotheses about the fuzzy expectation with respect to fuzzy data.

Based on a generalized metric for fuzzy numbers, Montenegro et al. (2001) study some two-(fuzzy)sample hypothesis tests for means concerning a fuzzy random variable in two populations.

Yao and $\mathrm{Wu}$ (2001) consider hybrid data which contain randomness and fuzziness, and give a fuzzy sequential test. They use the signed distance ranking to fuzzify the usual sequential test and obtain the fuzzy sequential test, and then use defuzzification to obtain the sequential test in the fuzzy sense.

Last et al. (1999), using a data mining approach, study fuzzy hypothesis testing and its application in medical diagnoses. See also Schenker et al. (2000). Grzegorzewski (2000) proposes a method for testing hypotheses with fuzzy data, which leads not to a binary decision but to a fuzzy decision result in which he defines a grade of acceptability for the null and the alternative hypotheses. See also Grzegorzewski (2002). Filzmoser and Viertl (2003) present an approach for testing hypotheses at the basis of fuzzy data, by introducing the fuzzy p-value. Taheri (2003) investigates the problem of testing of fuzzy hypotheses, and extend the sequential probability ratio test for such hypotheses.

For a review on the works on fuzzy hypotheses testing with Bayesian approach, see Section 6.

Finally, it should be mentioned that, some statisticians explain that, the precise hypotheses are not real hypotheses. They propose to consider some imprecise, but real, hypotheses. See, for example, Berger and Delampady (1987), and Berger et al. (1997).

\section{Regression}

In traditional statistical inference, as we know, the regression models are used frequently in the researches of the relations among several variables in a system. Observing some of the variables, we can make estimates and predictions for the others. If a system under consideration is not governed by random variables and/or crisp observation but is governed by possibility variables and/or imprecise observation, it is more natural to seek a fuzzy regression analysis for such a system. Fuzzy regression, in a general way, can be classified into two categories:

I) Fuzzy regression when the relations of the variables are subject to fuzziness, II) Fuzzy regression when the variables themselves are fuzzy.

With another point of view the works on fuzzy regression, in a general way, can be classified into two categories:

I) The possibilistic regression analysis based on possibility concepts (since membership functions of fuzzy sets are often described as possibility distributions, (Dubois and Prade, 1988), this approach is called possibilistic regression analysis),

II) Least squares method for minimizing errors for the estimated outputs.

The characterization of the above cases is developed from different perspectives, and thus there exist several conceptual and methodological approaches to fuzzy regression. It should be mentioned that, in some approaches more than one case is considered to provide a fuzzy regression model. In the following, we review some topics in this field. 
Fuzzy regression analysis was first proposed by Tanaka et al. $(1980,1982)$ where a fuzzy linear system is used as a regression model, (see also Tanaka, 1987; Tanaka et al., 1987). They consider a regression model in which the relation of the variables are subject to fuzziness, i.e., the model with crisp input and fuzzy parameters. Their approaches are developed in several ways, see for example, Peters (1994), Luczynski and Matloka (1995), Tanaka et al. (1995), Tanaka and Lee (1999), and Yen et al. (1999).

An application of the mentioned model to sale forecasting is discussed in Heshmaty and Kandel (1985). A modified form of possibilistic regression is proposed by Sakawa and Yano (1991).

It should be mentioned that, possibilistic regression usually leads to a mathematical programming problem. Bardossy (1990) introduce the general form of regression equations, and shows how the problem of fuzzy regression can be formulated as a mathematical programming problem.

Interval regression analysis is considered by Tanaka and Lee (1998). In addition, Wang and Tsaur (2000b) provide an insight into regression intervals so that regression interval analysis, data type analysis and variable selections can be analytically performed. Also, Entani and Tanaka (2000) extend the exponential possibility regression to the interval outputs.

Another direction of fuzzy regression is fuzzy least squares approach. This method is based on the notion of distance between the predicted fuzzy outputs and the observed fuzzy outputs and the goodness-of-fit. Diamond (1987) propose models for least squares fitting for crisp input fuzzy output and for fuzzy input-output where the distance of fuzzy numbers is defined to measure the best fit for models. Diamond's method has been revisited in Diamond and Korner (1997). Bardossy et al. (1992) define a new class of distance on fuzzy numbers, and consider the model involving fuzzy input and fuzzy parameters. $\mathrm{Xu}$ (1997) discusses the problem of least squares fitting of fuzzy-valued data by developing an special curve regression model for fitting this type of data.

Hong et al. (2001) consider a least-squares approach to the regression model with fuzzy-input and fuzzy parameters, using shape preserving operations. Chang and Ayyub (1997) develop a method for hybrid least squares regression, based on the weighted fuzzy arithmetic and the least squares fitting criterion. See also Chang (2001). Wang and Tsaur (2000a) propose a modified fuzzy least squares method for a crisp input fuzzy output model.

Fuzzy prediction based on regression models is studied by Yager (1982). In addition, Arnold and Stahlecker (1998) present an approach haw empirical data and fuzzy prior information may be combined for prediction purpose.

Jajuga (1986) present another approach which is useful in the case of heterogeneous observations.

Celmins (1987) deals with quadratic membership functions based on least squares fitting with indicators of discord, data spread dilator, etc. Nather and Korner (1998) extend classical estimates in the linear regression with crisp and fuzzy input and fuzzy output cases, with a least squares approach.

Now, let us review some specially works in fuzzy regression.

Wang and Li (1990), based on the concept of possibility variable, its distribution, and the independence among possibility variables, study the regression analysis of fuzzy 
valued variables.

Bardossy et al. (1992) define a new class of distance on fuzzy numbers and consider the model involving fuzzy input and fuzzy parameters.

Cheng and Lee (1999) propose a fuzzy adaptive network approach to fuzzy regression. They use radial basic function network in fuzzy regression analysis without predefined functional relationship between the input and output, as well (Cheng and Lee, 2001).

Sometimes it is hard to evaluate the goodness of fit of a fuzzy regression model. Toyoura and Watada (2000) propose two indices to evaluate a fuzzy regression model. In addition, Sadeghpour Gildeh and Gien (2002a) provide a goodness of fit index to evaluate the goodness of fit between the observed values and the estimated values in a fuzzy regression model.

D‘Urso and Gastaldi (2000) propose a doubly linear adaptive fuzzy regression model, based on two linear models: a core regression model and spread regression model, to explain the centers of the fuzzy observations and for their spreads.

Guo and Tanaka (2001) propose a fuzzy DEA (data envelopment analysis) model to deal with the efficiency evaluation problem with the given fuzzy input and output data. They extend their model with considering the relationship between DEA and regression analysis.

Peters (2001) present a forecasting model based on fuzzy pattern recognition and weighted linear regression. In this model fuzzy pattern recognition is used to find homogeneous fuzzy classes in a heterogeneous data set, and then for each class a weighted regression analysis is conducted. In addition, the forecasting results obtained by the class regression analysis are aggregated to obtain the overall estimation of the regression model. Roychowdhury and Pedrycz (2002) attempt to combine regression and fuzzy rules to model temporal systems.

Arnold and Gerke (2003) provide a method of testing fuzzy linear hypotheses in the linear regression models.

Finally, it should be mentioned that, Reden and Woodall (1994) review and examine some approaches to fuzzy regression and discuss their strength and weakness relative to each other.

Kim et al. (1996), discuss and contrast the characteristics of statistical linear regression and fuzzy linear regression, in terms of basic assumption, parameter estimation, and applications.

\section{Bayes Methods}

There are a lot of researches regarding Bayesian methods combined with fuzzy set theory. Some works are in the context of statistics. We review some of them in this section.

First, it should be mentioned that, Viertl (1987) explains the necessity of developing a fuzzy Bayesian inference. See also Walley (1991) for some brief discussions on ordinary and fuzzy decision analysis.

Tanaka et al. (1979), and Uemura (1991, 1993a,b) formulate the fuzzy-Bayes decision rule to facilitate determination of the loss function of a Bayes decision rule in a fuzzy environment. 
Buckley $(1983 a, b)$ investigates some problems of statistical inference with fuzzy data, in a fuzzy decision making problem.

Gil et al. (1985a) extend the Bayesian method for the point estimation when the available information is fuzzy.

Bayes formula for fuzzy probability measure is studied by Piasecki (1986, 1987), and, in a more general manner, by Mesiar and Piasecki (1990).

Viertl and Hule (1991) generalize the Bayes' theorem to the case of fuzzy data. They extend the concept of HPD-regions to this case. In addition, based on a generalized integration concept for functions with fuzzy values, Viertl (1999) study the Bayes theorem, confidence regions, and predictions for fuzzy prior distribution and fuzzy data. See also Viertl and Hareter (2002).

Schnatter (1993) combines Bayes procedure and fuzzy set theory to provide some methods both for samples of fuzzy data and for prior distributions with non-precise parameters.

Dubois and Prade (1997) introduce a Bayesian conditioning operation in possibility theory, adapted to the idea of focusing on a body of knowledge for a reference class described by some evidences.

A Bayes and minimax procedure for testing simple hypotheses is given by Casals et al. (1986a,b). They consider the problem of testing the ordinary (crisp) statistical hypotheses when the observations do not provide exact but rather fuzzy information. Delgado et al. (1985) consider the problem of fuzzy hypotheses testing with ordinary data. Casals (1993) works on the same problem but with fuzzy observations, in the context of fuzzy decision problem (Tanaka et al., 1979).

Taheri and Behboodian (2001) study the problem of hypotheses testing, from a Bayesian point of view, when the observations are ordinary (crisp) and the hypotheses are fuzzy. They extend their approach to the case of fuzzy observations and fuzzy hypotheses (Taheri and Behboodian, 2002).

Lapiga and Polyakov (1992) use statistical modeling of membership functions to diminish subjectivity and to determine the membership function analytical forms in fuzzy decision-making.

Gertner and Zhu (1997), based on two extensions of likelihood functions, have generalized Bayesian estimates for use when sample information and prior distribution are fuzzy. They apply their method to forest surveys.

Bayesian analysis of a decision problem with fuzzy-valued utilities or losses is studied by Lopez-Diaz and Gil (1998). They consider and illustrate the Bayesian analysis of estimation and hypotheses testing problems, as some special cases.

Bayesian fuzzy kriging is studied by Bandemer and Gebhardt (2000). They combine two approaches generalizing the usual kriging technique for prediction in fields: the Bayesian approach incorporating prior knowledge and the fuzzy set approach reflecting imprecise observations.

Lapointe and Bobee (2000) develop the counterpart of the Bayes' rule in the possibilistic framework with the use of conditional possibility distributions. 


\section{Some Other Fields}

There are some studies on fuzzy statistical information theory. For example Menendez et al. (1992) extend the Jensen difference divergence measure and define two information measures to compare statistical experiments when the available information is not exact. They work on sufficiency of a fuzzy information system, as well (Menendez et al., 1989). Yen and Wang (1998) propose several information theoretic optimal fuzzy models construction by extending some statistical information criteria. See also Menendez (1998) for another approach in this subject. Lubiano et al. (1999), and Sadeghpour Gildeh and Gien (2002b) study the Rao-Blackwell type theorem for fuzzy random variables.

Fuzzy expected value (FEV) is introduced by Kandel and Byatt (1978). Properties of FEV is studied by Schnider and Kandel (1988a,b). Also, they proceed to show how to approximate the FEV in a fuzzy environment by introducing the fuzzy expected interval, and study some applications to the management of uncertainty in fuzzy expert systems (Schnider and Kandel, 1988b, 1993). Using FEV, Schnider and Craig (1992) introduce a method for histogram equalization.

Viertl (1989) consider the estimation of the reliability function when observed data is fuzzy. Niculescu and Viertl (1992) study the reliability models with fuzzy data and compare two fuzzy sample mean estimators, in this field. Wu (1997) studies the system reliability by considering the failed or functioning probability of each component in the system as nonnegative fuzzy numbers under the definition of fuzzy-valued probability measure. Dunyak et al. (1999) study the system reliability based on the fuzzy probability, too. It should be mentioned that, some works have been done on possibilistic reliability analysis, see for example Cappelle and Kerre (1993, 1997).

The extended methods in the construction of control charts for fuzzy observations are proposed by Wang and Raz (1990), and Kanagava et al. (1993). In this field, see also Woodall et al. (1997).

Peizhuang and Xihui (1984) establish the concept of the set-valued statistics on the basis of the theory of random sets, and Sizhong (1987) extend this concept to fuzzy setvalued statistics. On the basis of the random intervals, Xihe (1989) demonstrate stability of membership frequency in a proper mathematical model.

Bergh and Berg (2000) introduce the competitive fuzzy exception learning algorithm based on fuzzy frequency distributions. See also Berg et al. (2001). Bodjanova (2000) suggests a method for constructing a generalized histogram displaying the distribution of a sample of fuzzy numbers into some fuzzy intervals. Some discussions on descriptive statistics with fuzzy data, are presented by Kruse and Meyer (1987), and by Viertl (1996).

A couple of works have been done on the sampling techniques, with non-precise data. Lopez-Diaz and Gil (1998) define a fuzzy unbiased estimator of the sample mean in random sampling with replacement from a finite population. See also Lubiano and Gil (1999). Garcia et al. (2001) illustrate estimating the expected value of fuzzy random variables in the stratified random sampling.

Schnatter (1991) discusses how fuzziness of data is propagated when statistical inference for samples of fuzzy data is carried out. She illustrates how methods from descriptive statistics can be generalized for fuzzy data (Schnatter, 1992).

Manton et al. (1991, 1994), describe and illustrate the use of fuzzy partition statistical 
methods, as a means of analyzing high dimensional data with categorical responses.

Some methods of statistical inference with fuzzy data, are reviewed by Viertl (2002a). For a review in more details, see Viertl (2002b).

Behboodian and Mohammadpour (2002) consider a distribution depends on some unknown crisp parameters and a fuzzy parameter with a known membership function. They present a method for estimation of the fuzzy parameter, and a procedure for hypothesis testing about one of the unknown crisp parameters.

Fuzzy order statistics and its applications, specially in fuzzy clustering, is studied by Kersten (1999).

Chen $(1995,2000)$ introduces a general framework of fuzzy analysis of statistical evidence methodologies for pattern classification and knowledge discovery. His method is based on the possibility measure, which does not require a precise belief model and, in a sense, it includes the Bayesian classifiers as special case.

Chen et al. (2000) combine fuzzy methods and statistical methods of ANOVA and factor analysis to propose a method for objective evaluation of fabric softness.

Rojas et al. (1999) study the relevancy and relative importance of the operators, specially defuzzifiers and T-norms, involved in the fuzzy inference process.

Fuzzy spatial statistics, which is usually referred to as geostatistics, is proposed by Lee (1995). He suggests neural learning combined with fuzzy representation for handling the variogram, which is essentially a covariance correlation, and the kriging, which is an unbiased method for estimating the missing data (Lee, 2000). Burrough (2001) shows that fuzzy set theory is a useful tool for spatial analysis when probabilistic approaches are inappropriate or impossible.

\section{Acknowledgment}

The author would like to thank A. Rejali and R. Viertl for their numerous helpful suggestions.

\section{References}

B.F. Arnold. Statistical tests optimally meeting certain fuzzy requirements on the power function and on the sample size. Fuzzy Sets Syst., 75(2):365-372, 1995.

B.F. Arnold. An approach to fuzzy hypothesis testing. Metrika, 44:119-126, 1996.

B.F. Arnold. Testing fuzzy hypothesis with crisp data. Fuzzy Sets Syst., 94(2):323-333, 1998.

B.F. Arnold and O. Gerke. Testing fuzzy linear hypotheses in linear regression models. Metrika, 57:81-95, 2003.

B.F. Arnold and P. Stahlecker. Prediction in linear regression combining crisp data and fuzzy prior information. Statistics and Decisions, 16:19-33, 1998. 
H. Bandemer and A. Gebhardt. Bayesian fuzzy kriging. Fuzzy Sets Syst., 112:405-418, 2000.

A. Bardossy. Note on fuzzy regression. Fuzzy Sets Syst., 37:65-75, 1990.

A. Bardossy, R. Hagaman, L. Duckstein, and I. Bogardi. Fuzzy least-squares regression: theory and applications. In J. Kacprzyk and M. Fedrizzi, editors, Fuzzy Regression Analysis, pages 181-193. Physica-Verlag, Heidelberg, 1992.

J. Behboodian and A. Mohammadpour. Estimation of a fuzzy parameter and its application in hypothesis testing. In R. Borzouee, editor, Proc. of the Third Seminar on Fuzzy Sets and Its Applications, pages 23-45. Univ. of Zahedan and Baluchestan, Zahedan, Iran, 2002.

J. Berg, W.M. Bergh, and U. Keymak. Probabilistic and statistical fuzzy set foundations of competitive exception learning. In Proc. of the 10-th IEEE Int. Conf. Fuzzy Systems, pages 1035-1038. Melbourne, 2001.

J.O. Berger, B. Boukai, and Y. Wang. Unified frequentist and Bayesian testing of a precise hypothesis. Stat. Sci., 12:133-160, 1997.

J.O. Berger and M. Delampady. Testing precise hypotheses. Stat. Sci., 2:317-352, 1987.

W.M. Bergh and J. Berg. Competitive exception learning using fuzzy frequency distributions. Neural Network World, 10:59-71, 2000.

S. Bodjanova. A generalized histogram. Fuzzy Sets Syst., 116:155-166, 2000.

J.J. Buckley. Decision making under risk: a comparison of Bayesian and fuzzy set method. Risk Analysis, 3:157-168, 1983a.

J.J. Buckley. Fuzzy decision making with data: applications to statistics. Fuzzy Sets Syst., 16:139-147, 1983b.

P.A. Burrough. GIS and geostatistics: essential partners for spatial analysis. Environmental and Ecological Statistics, 8:361-377, 2001.

K.Y. Cai. Parameter estimation of normal fuzzy variables. Fuzzy Sets Syst., 55:179-185, 1993.

K.Y. Cai, C.Y. Wen, and M.L. Zhang. Fuzzy variables as a basis for a theory of fuzzy reliability in the possibility context. Fuzzy Sets Syst., 42:145-172, 1991.

B. Cappelle and E.E. Kerre. On a possibilistic approach to reliability theory. In Proc. of the Second International Symposium on Uncertainty Modelling and Analysis, pages 415-418. IEEE Computer Society Press, Univ. of Maryland, 1993.

B. Cappelle and E.E. Kerre. On the computability of possibilistic reliability. In B.M. Ayyoub and M.M. Gupta, editors, Uncertainty Analysis in Engineering and Sciences, pages 325-337. Kluwer, Boston, 1997. 
M.R. Casals. Bayesian testing of fuzzy parametric hypotheses from fuzzy information. RAIRO, Operations Research, 27:189-199, 1993.

M.R. Casals and M.A. Gil. A note on the operativeness of Neyman-Pearson tests with fuzzy information. Fuzzy Sets Syst., 30:215-220, 1989.

M.R. Casals, M.A. Gil, and P. Gil. On the use of Zadeh's probabilistic definition for testing statistical hypotheses from fuzzy information. Fuzzy Sets Syst., 20:175-190, 1986a.

M.R. Casals, M.A. Gil, and P. Gil. The fuzzy decision problem: An approach to the problem of testing statistical hypotheses with fuzzy information. Euro. J. Oper. Res., 27:71-382, 1986b.

A. Celmins. Least squares model fitting to fuzzy vector data. Fuzzy Sets Syst., 22:260269, 1987.

Y.O. Chang. Hybrid fuzzy least-squares regression analysis and its reliability measures. Fuzzy Sets Syst., 119:225-246, 2001.

Y.O. Chang and B.M. Ayyub. Hybrid least-squares regression analysis. In B.M. Ayyoub and M.M. Gupta, editors, Uncertainty Analysis in Engineering and Sciences, pages 179-191. Kluwer, Boston, 1997.

Y. Chen, B. Collier, P. Hu, and D. Quebedeaux. Objective evaluation of fabric softness. Textile Res. J., 70:443-448, 2000.

Y.Y. Chen. Statistical inference based on the possibility and belief measures. Trans. Amer. Math. Soc., 347:1855-1863, 1995.

Y.Y. Chen. Fuzzy analysis of statistical evidence. IEEE Trans. on Fuzzy Systems, 8: 796-799, 2000.

C.B. Cheng and E.S. Lee. Applying fuzzy adaptive network to fuzzy regression analysis. Comput. Math. Appl., 38:123-140, 1999.

C.B. Cheng and E.S. Lee. Fuzzy regression with radial basis function network. Fuzzy Sets Syst., 119:291-301, 2001.

N. Corral and M.A. Gil. The minimum inaccuracy fuzzy estimation: an extension of the maximum likelihood principle. Stochastica, 8:63-81, 1984.

N. Corral and M.A. Gil. A note on interval estimation with fuzzy data. Fuzzy Sets Syst., 28:209-215, 1988.

D.I. Dale. Probability, vague statements, and fuzzy sets. Philos. Science, 47:38-55, 1980.

M. Delgado, J.L. Verdegay, and M.A. Vila. Testing fuzzy-hypotheses, a Bayesian approach. In M.M. Gupta, editor, Approximate Reasoning in Expert Systems, pages 307316. North-Holland, Amsterdam, 1985. 
P. Diamond. Least squares fitting of several fuzzy variables. In Proc. of Second IFSA Congress, pages 20-25. IFSA, Tokyo, 1987.

P. Diamond and R. Korner. Extended fuzzy linear models and least square estimates. Comput. Math. Appl., 33:15-32, 1997.

D. Dubois and H. Prade. Fuzzy sets and probability: misunderstandings, bridges, and gaps. In Proc. of the Second IEEE International Conference on Fuzzy Systems, pages 1059-1068. IEEE, Piscataway, 1993.

D. H. Dubois and Prade. Fuzzy Sets and Statistical Possibility Theory. Plenum Press, New York, 1988.

P. Dubois and H. Prade. Fuzzy sets and statistical data. Euro. J. Oper. Res., 25:345-356, 1986.

P. Dubois and H. Prade. Bayesian conditioning in possibility theory. Fuzzy Sets Syst., 92: 223-240, 1997.

J. Dunyak, I.W. Saad, and D. Wunsch. A theory of independent fuzzy probability for system reliability. IEEE Trans. on Fuzzy Systems, 7:286-294, 1999.

P. D‘Urso and T. Gastaldi. A least-squares approach to linear regression analysis. Comp. Stat. and Data Anal., 34:427-440, 2000.

T. Entani and H. Tanaka. Exponential possibility regression with interval outputs. In Proc. of the Fourth Asian Fuzzy Systems Symposium, pages 100-104. Tsukuba, Japan, 2000.

P. Filzmoser and R. Viertl. Testing hypotheses with fuzzy data: the fuzzy p-value. Metrika (to appear), 2003.

D. Garcia, M.A. Lubiano, and C. Alonso. Estimating the expected value of fuzzy random variables in the stratified random sampling from finite populations. Inform. Sci., 138: 165-184, 2001.

G.Z. Gertner and H. Zhu. Bayesian estimation in forest surveys when samples or prior information are fuzzy. Fuzzy Sets Syst., 77:277-290, 1997.

M.A. Gil. Fuzziness and loss of information in statistical problems. IEEE Trans. on Systems Man and Cybernet. SMC, 17:1012-1025, 1987.

M.A. Gil. A note on the connection between fuzzy numbers and random intervals. Stat. Prob. Lett., 13:311-319, 1992.

M.A. Gil, N. Corral, and P. Gil. The fuzzy decision problem: an approach to the point estimation problem with fuzzy information. Euro. J. Oper. Res., 22:26-34, 1985a.

M.A. Gil, N. Corral, and P. Gil. The minimum inaccuracy estimates in $\chi^{2}$ tests for goodness of fit with fuzzy observations. J. Stat. Plan. Inf., 19:95-115, 1985b. 
M. Grabisch, H.T. Nguyen, and E. Walker. Fundamentals of Uncertainty Calculi with Applications to Fuzzy Inference. Kluwer, Dordrecht, 1995.

P. Grzegorzewski. Testing statistical hypotheses with vague data. Fuzzy Sets Syst., 112: 501-510, 2000.

P. Grzegorzewski. Testing fuzzy hypotheses with vague data. In C. Bertoluzzi, editor, Statistical Modeling, Analysis and Management of Fuzzy Data, pages 213-225. Physica-Verlag, Heidelberg, 2002.

P. Guo and H. Tanaka. Fuzzy DEA: a perceptual evaluation method. Fuzzy Sets Syst., 119:149-160, 2001.

B. Heshmaty and A. Kandel. Fuzzy linear regression and its application to forecasting in uncertain environment. Fuzzy Sets Syst., 15:159-191, 1985.

H. Hisdal. Are grades of membership probabilities? Fuzzy Sets Syst., 25:325-348, 1988.

D.H. Hong, J. Song, and H.Y. Do. Fuzzy least-squares linear regression analysis using shape preserving operations. Inform. Sci., 138:185-193, 2001.

C. Hwang and J. Yao. Independent fuzzy random variables and their application. Fuzzy Sets Syst., 82:335-350, 1996.

K. Jajuga. Linear fuzzy regression. Fuzzy Sets Syst., 20:343-353, 1986.

A. Kanagava, F. Tamaki, and H. Ohta. Control charts for process average and variability based on linguistic data. Inter. J. of Production Research, 2:913-922, 1993.

A. Kandel and W.J. Byatt. Fuzzy sets, fuzzy algebra, and fuzzy statistics. Proc. of The IEEE, 66(12):1619-1639, 1978.

P.R. Kersten. Fuzzy order statistics and their application to fuzzy clustering. IEEE Trans. on Fuzzy Systems, 7:708-712, 1999.

K.J. Kim, H. Moskowitz, and M. Koksalan. Fuzzy versus statistical linear regression. Euro. J. Oper. Res., 92:417-434, 1996.

G.J. Klir. Is there more to uncertainty than some probability theorists might have us believe? Int. J. General Systems, 15:347-378, 1989.

R. Korner. An asymptotic $\alpha$-test for the expectation of random fuzzy variables. J. Stat. Plan. Inf., 83:331-346, 2000.

B. Kosko. Fuzziness vs. probability. Inter. J. of General Systems, 17:211-240, 1990.

R. Kruse. Statistical estimation with linguistic data. Inform. Sci., 33:197-207, 1984.

R. Kruse and K.D. Meyer. Statistics with Vague Data, volume 33. Reidel, Dordrecht, 1987. 
A.G. Lapiga and V.V. Polyakov. On statistical methods in fuzzy decision-making. Fuzzy Sets Syst., 47:303-311, 1992.

S. Lapointe and B. Bobee. Revision of possibility distributions: a Bayesian inference pattern. Fuzzy Sets Syst., 116:119-140, 2000.

M. Last, A. Schenker, and A. Kandel. Applying fuzzy hypothesis testing to medical data. In N. Zhong et al., editor, New Directions in Rough Sets, Data Mining, and Granular-Soft Computing, Lecture Notes in Artificial Intelligence Series, Vol. 1711, pages 221-229. Springer, Berlin, 1999.

M. Laviolette and J.W. Seaman. Evaluating fuzzy representations of uncertainty. The Mathematical Scientist, 17:26-41, 1992.

M. Laviolette and J.W. Seaman. Unity and diversity of fuzziness - from a probability viewpoint. IEEE Trans. on Fuzzy Systems, 2:38-42, 1994.

M. Laviolette, J.W. Seaman, J.D. Barrett, and W.H. Woodall. A probabilistic and statistical view of fuzzy methods (with discussion). Technometrics, 37:249-296, 1995.

E.S. Lee. Fuzzy spatial statistics. In Selected Papers of Engineering Chemistry and Metallurgy, pages 151-157. Institute of Chemical Metallurgy, Chinese Academy of Science, China, 1995.

E.S. Lee. Neuro-fuzzy estimation in spatial statistics. J. Math. Anal. Appl., 249:221-231, 2000.

M. Lopez-Diaz and M.A. Gil. Reversing the order of integration in iterated expectations of fuzzy random variables, and statistical applications. J. Stat. Plan. Inf., 74:11-29, 1998.

M.A. Lubiano and M.A. Gil. Estimating the expected value of fuzzy random variables in random sampling from finite populations. Statistical Papers, 40:277-295, 1999.

M.A. Lubiano, M.A. Gil, and M. Lopez-Diaz. On the Rao-Blackwell Theorem for fuzzy random variables. Kybernetika, 35:167-175, 1999.

W. Luczynski and M. Matloka. Fuzzy regression models and their applications. J. Fuzzy Math., 3:583-589, 1995.

K.G. Manton, E. Stallard, and M.A. Woodbury. A multivariate event history based upon fuzzy states: estimation from longitudinal surveys with informative nonresponse. $J$. Official Statistics, 7:261-293, 1991.

K.G. Manton, M.A. Woodbury, and D.H. Tolley. Statistical Applications Using Fuzzy Sets. Wiley, New York, 1994.

R.A. McCain. Fuzzy confidence intervals. Fuzzy Sets Syst., 10:281-290, 1983. 
M.L. Menendez. The minimum $\phi$-divergence estimates with fuzzy observations: statistical applications. Fuzzy Sets Syst., 96:101-109, 1998.

M.L. Menendez, J.A. Pardo, and L. Pardo. Sufficient fuzzy information systems. Fuzzy Sets Syst., 32:97-105, 1989.

M.L. Menendez, J.A. Pardo, and L. Pardo. Some statistical applications of generalized jensen difference divergence measures for fuzzy information systems. Fuzzy Sets Syst., 52:169-180, 1992.

R. Mesiar and K. Piasecki. On a possible generalization of the Bayes method of inference. Fuzzy Sets Syst., 37:351-357, 1990.

M. Montenegro, M.R. Casals, M.A. Lubiano, and M.A. Gil. Two-sample hypothesis tests of a fuzzy random variable. Inform. Sci., 133:89-100, 2001.

S. Nahmias. Fuzzy variables. Fuzzy Sets Syst., 1:97-100, 1978.

W. Nather and R. Korner. Linear regression with random fuzzy numbers. In B.M. Ayyub and M.M. Gupta, editors, Uncertainty Analysis in Engineering and Sciences, pages 193-211. Kluwer, Boston, 1998.

S.P. Niculescu and R. Viertl. A comparison between two fuzzy estimators for the mean. Fuzzy Sets Syst., 48:341-350, 1992.

T. Okuda. A statistical treatment of fuzzy observations: estimation problems. In Preprints of Second IFSA Congress, pages 51-55. IFSA, 1987.

M.G.A. Paris. Nearly ideal binary communication in squeezed channels. Physical Review A, 64:14304-14308, 2001.

W. Peizhuang and L. Xihui. Set-valued statistics. J. Engineering Math., 1, 1984.

G. Peters. Fuzzy linear regression with fuzzy intervals. Fuzzy Sets Syst., 63:45-55, 1994.

G. Peters. A linear forecasting model and its application to economic data. J. Forecast., 20:315-328, 2001.

K. Piasecki. On the Bayes formula for fuzzy probability measures. Fuzzy Sets Syst., 18: 183-185, 1986.

K. Piasecki. Note to Piasecki (1986). Fuzzy Sets Syst., 24:121-122, 1987.

D. Reden and W. Woodall. Properties of certain fuzzy linear regression methods. Fuzzy Sets Syst., 64:361-375, 1994.

I. Rojas, J. Ortega, F.J. Pelayo, and A. Prieto. Statistical analysis of the main parameters in the fuzzy inference process. Fuzzy Sets Syst., 102:157-173, 1999.

C. Romer and A. Kandel. Statistical tests for fuzzy data. Fuzzy Sets Syst., 72:1-26, 1995. 
S. Roychowdhury and W. Pedrycz. Modeling temporal functions with granular regression and fuzzy rules. Fuzzy Sets Syst., 126:377-387, 2002.

J.J. Saade and H. Schwarzlander. Fuzzy hypothesis testing with hybrid data. Fuzzy Sets Syst., 35:197-212, 1990.

B. Sadeghpour Gildeh and D. Gien. A goodness of fit index to reliability analysis in fuzzy model. In A. Grmela, editor, Advances in Intelligent Systems, Fuzzy Systems, Evolutionary Computation, pages 78-83. WSEAS Press, Greece, 2002a.

B. Sadeghpour Gildeh and D. Gien. $d_{p, q}$-distance and Rao-Blackwell Theorem for fuzzy random variables. In Proc. of the 8th International Conference on Fuzzy Theory and Technology. Durham, USA, 2002b.

M. Sakawa and H. Yano. Moltiobjective fuzzy linear regression analysis for fuzzy data. Fuzzy Sets Syst., 47:173-181, 1991.

A. Schenker, M. Last, and A. Kandel. Fuzzy hypotheses testing: verification-based data mining. Manuscript, 2000.

S. Schnatter. On propagation of fuzziness of data. Environmetrics, 2:241-252, 1991.

S. Schnatter. On statistical inference for fuzzy data with applications to descriptive statistics. Fuzzy Sets Syst., 50:143-165, 1992.

S. Schnatter. On fuzzy Bayesian inference. Fuzzy Sets Syst., 60:41-58, 1993.

M. Schnider and M. Craig. On the use of fuzzy sets in histogram equalization. Fuzzy Sets Syst., 45:271-278, 1992.

M. Schnider and A. Kandel. Expectations in fuzzy environments. J. Fuzzy Logic and Intelligent Systems, 3:76-93, 1993.

S. Schnider and A. Kandel. Properties of the fuzzy expected value and the fuzzy expected interval. Fuzzy Sets Syst., 26:373-385, 1988a.

S. Schnider and A. Kandel. Properties of the fuzzy expected value and the fuzzy expected interval in fuzzy environment. Fuzzy Sets Syst., 28:55-68, 1988 b.

G. Sizhong. Fuzzy random set and fuzzy set-valued statistics. In Preprints of Second IFSA Congress, pages 20-25. IFSA, Tokyo, 1987.

J.C. Son, I. Song, and H.Y. Kim. A fuzzy decision problem based on the generalized Neyman-Pearson criterion. Fuzzy Sets Syst., 47:65-75, 1992.

W. Stallings. Fuzzy set theory versus Bayesian statistics. IEEE Trans. on Systems Man and Cybernet. SMC, 7:216-219, 1977.

S.M. Taheri. SPRT for non-precise hypotheses. Manuscript, 2003. 
S.M. Taheri and J. Behboodian. Neyman-Pearson Lemma for fuzzy hypotheses testing. Metrika, 49:3-17, 1999.

S.M. Taheri and J. Behboodian. A Bayesian approach to fuzzy hypotheses testing. Fuzzy Sets Syst., 123:39-48, 2001.

S.M. Taheri and J. Behboodian. Fuzzy hypotheses testing with fuzzy data: a Bayesian approach. In R.P. Nikhil and M. Sugeno, editors, Advances in Soft Computing, AFSS2002, pages 527-533. Springer, Berlin, 2002.

S. Takayanagi and N. Cliff. The fuzziness index for examining human statistical decisionmaking. In R.J. Marks, editor, Fuzzy Logic Technology and Applications, pages 509514. IEEE Technical Activities Board, 1994.

H. Tanaka. Fuzzy data analysis by possibilistic linear models. Fuzzy Sets Syst., 24:363375, 1987.

H. Tanaka, I. Hayashi, and J. Watada. Possibilistic linear regression analysis based on possibility measure. In Preprints of Second IFSA Congress, pages 317-320. IFSA, Tokyo, 1987.

H. Tanaka, H. Ishibuchi, and S. Yoshikawa. Exponential possibility regression analysis. Fuzzy Sets Syst., 69:305-318, 1995.

H. Tanaka and H. Lee. Exponential possibility regression analysis by identification method of possibilistic coefficients. Fuzzy Sets Syst., 106:155-165, 1999.

H. Tanaka, T. Okuda, and K. Asai. Fuzzy information and decision in statistical model. In M.M. Gupta et al., editor, Advances in Fuzzy Set Theory and Applications, pages 303-320. North-Holland, Amsterdam, 1979.

H. Tanaka, S. Uejima, and K. Asai. Fuzzy linear regression model. IEEE Trans. Systems Man Cybernet., 10:2933-2938, 1980.

H. Tanaka, S. Uejima, and K. Asai. Linear regression analysis with fuzzy model. IEEE Trans. Systems Man Cybernet., 12:903-907, 1982.

Y. Toyoura and J. Watada. Evaluation of fuzzy regression analysis. In Proc. of the Fourth Asian Fuzzy Systems Symposium, pages 1015-1020. Tsukuba, Japan, 2000.

Y. Uemura. A decision rule on fuzzy events. Japanese J. Fuzzy Theory and Systems, 3: 291-300, 1991.

Y. Uemura. A decision rule on fuzzy events under an observation. J. of Fuzzy Math., 1: 39-52, 1993a.

Y. Uemura. A simple decision rule on fuzzy events. Cybernet. Syst. Int. J., 24:509-521, 1993b. 
R. Viertl. Is it necessary to develop a fuzzy Bayesian inference? In R. Viertl, editor, Probability and Bayesian Statistics. Plenum Press, New York, 1987.

R. Viertl. Estimation of the reliability function using fuzzy life time data. In P.K. Bose et al., editor, Quality for Progress and Development. Wiley Eastern, New Delhi, 1989.

R. Viertl. Statistical Methods for Non-Precise Data. CRC Press, Boca Raton, 1996.

R. Viertl. Statistics and integration of fuzzy functions. Environmetrics, 10:487-491, 1999.

R. Viertl. Statistics with one-dimensional fuzzy data. In C. Bertoluzzi et al., editor, Statistical Modeling, Analysis and Management of Fuzzy Data, pages 199-212. PhysicaVerlag, Heidelberg, 2002a.

R. Viertl. Statistical inference with non-precise data. In Encyclopedia of Life Support Systems. UNESCO, Paris, 2002b. On-line: www.eolss.unesco.org.

R. Viertl and D. Hareter. Bayes theorem for non-precise a-priori distribution and nonprecise data. Manuscript, 2002.

R. Viertl and H. Hule. On Bayes' theorem for fuzzy data. Statistical Papers, 32:115-122, 1991.

P. Walley. Statistical Reasoning with Imprecise Probabilities. Chapman and Hall, London, 1991.

J.H. Wang and T. Raz. On the construction of control charts using linguistic variables. Inter. J. Production Research, 28:477-487, 1990.

J.H. Wang and R.C. Tsaur. Resolution of fuzzy regression model. Euro. J. Oper. Re., 126: 637-650, 2000a.

J.H. Wang and R.C. Tsaur. Insight of a fuzzy regression model. Fuzzy Sets Syst., 112: 355-369, 2000b.

Z. Wang and S. Li. Fuzzy linear regression analysis of fuzzy valued variables. Fuzzy Sets Syst., 36:125-136, 1990.

N. Watanabe. Fuzzy random variables and statistical inference. Japanese J. of Fuzzy Theory and Systems, 8:833-846, 1996.

N. Watanabe and T. Imaizumi. A fuzzy statistical test of fuzzy hypotheses. Fuzzy Sets Syst., 53:167-178, 1993.

W.H. Woodall, K.L. Tsui, and G.R. Tucker. A review of statistical and fuzzy control based on categorical data. In Frontiers in Statistical Quality Control, pages 83-89. Physica-Verlag, Heidelberg, 1997.

H. Wu. Fuzzy reliability analysis based on fuzzy numbers. Inform. Sci., 103:135-159, 1997. 
L. Xihe. Stability of random membership frequency and fuzzy statistics. Fuzzy Sets Syst., 29:89-102, 1989.

R. Xu. S-Curve regression model in fuzzy environment. Fuzzy Sets Syst., 90:317-326, 1997.

R.R. Yager. Fuzzy prediction based on regression models. Inform. Sci., 26:45-63, 1982.

J. Yao and C. Hwang. Point estimation for $\mathrm{n}$ sizes of random sample with one vague data. Fuzzy Sets Syst., 80:205-215, 1996.

J. Yao and K. Wu. Sequential test for fuzzy hypothesis based on hybrid data and signed distance ranking. Manuscript, 2001.

J. Yen and L. Wang. Applications of statistical information criteria for optimal fuzzy model construction. IEEE Trans. on Fuzzy Systems, 6:362-372, 1998.

K.K. Yen, S. Ghoshray, and G. Roig. A linear regression model using triangular fuzzy number coefficients. Fuzzy Sets Syst., 106:167-177, 1999.

L.A. Zadeh. Probability and fuzziness are completibility rather than contradictory. Technometrics, 37:271-277, 1995.

L.A. Zadeh. Toward a perception-based theory of probabilistic reasoning with imprecise probabilities. J. Stat. Plan. Inf., 105:233-264, 2002.

Author's address:

Univ. Asso. Prof. Dr. S. Mahmoud Taheri

School of Mathematical Sciences

Isfahan University of Technology

Isfahan, 84154

Iran

Tel. +983113913198

Fax +98 3113912602

E-mail: Taheri@cc.iut.ac.ir

http://www.iut.ac.ir/math/ 